\title{
Practical lessons for creating affordance-based interventions for sustainable behavior change
}

\author{
Kaaronen, Roope Oskari $* 1,2,3$; Rietveld, Erik ${ }^{3,4,5,6}$. \\ 1. Faculty of Biological and Environmental Sciences, University of Helsinki, Helsinki, Finland \\ 2. Helsinki Institute of Sustainability Science, University of Helsinki, Helsinki, Finland \\ 3. University of Amsterdam, Academic Medical Center, Amsterdam, The Netherlands \\ 4. RAAAF, Amsterdam, The Netherlands \\ 5. Department of Philosophy/ILLC, University of Amsterdam, Amsterdam, The Netherlands \\ 6. Department of Philosophy, University of Twente, Enschede, The Netherlands
}

*Corresponding author. Email: roope.kaaronen@helsinki.fi

This is the authors' original manuscript (preprint) version.

The published version of this article is available at One Earth, vol. 4 issue 10: https://doi.org/10.1016/j.oneear.2021.09.013

\begin{abstract}
Radical collective behavior change is required to develop sustainable forms of urban life. This demands redesign of everyday environments. However, the ways in which our material world shape our behaviors are still understudied and underappreciated. Not much is known about how collective behaviors are facilitated through infrastructural or material interventions. Here, we draw upon 15 years of experience at RAAAF, an Amsterdam-based collective for visual art and architecture, to introduce ten practical lessons for developing strategic design interventions for affordance-based behavior change in urban environments. Affordances are the possibilities for action provided by the environment. Strategic design interventions aim to set collective social change in motion by developing sustainable affordances and dismantling unsustainable behavioral constraints. Strategic design interventions seek to inspire policies and public imagination. Whereas scientific studies aim to describe reality as it is, RAAAF's material interventions help imagine how the shared urban environment could be in the future.
\end{abstract}

Keywords: behavior change, sustainability, affordance, design, architecture, nudge, practical knowledge, climate change, social tipping points 


\section{Introduction}

An overwhelming scientific consensus suggests that major emission reductions in multiple domains are required to reach sustainable modes of human existence on Earth ${ }^{1-3}$. This will undoubtedly require drastic change in the ways in which humans behave ${ }^{4,5}$. This is particularly the case within urban environments, where $68 \%$ of the world population are projected to live in by $2050{ }^{6}$, in what will likely be the largest human migration event ever ${ }^{7}$. If current trends continue, more urban areas will be built during the first three decades of the $21^{\text {st }}$ century than in the totality of preceding human history ${ }^{8}$. According to some estimates, urban areas already currently generate ca. $90 \%$ of global economic activity and up to $70 \%$ of global greenhouse gas emissions ${ }^{9}$, and are key drivers of exponential consumption of natural resources ${ }^{7,10}$. If societies worldwide are to develop within planetary boundaries ${ }^{2,11}$, the ongoing urban migration cannot go hand-in-hand with the historical trend of growth of energy and resource consumption during urbanization and industrialization processes ${ }^{1,7,12}$.

This presents us with the grand challenge of collective sustainable behavior change in urban environments. The growing threat of abrupt and irreversible adverse tipping points in climate change ${ }^{13}$ and other Earth Systems ${ }^{3}$ suggest that radical, nonlinear and collective change in human behavior is required to counter these processes. This nonlinear assumption is often embodied in models of sustainable development, which assume behavior change (and, e.g., related clean technology adoption or emission reduction) to follow a roughly S-shaped timeline, where societies reach self-reinforcing changes in collective behavior patterns, eventually settling down in a new sustainable state ${ }^{1,14-16}$. In practice, achieving such a transition requires the triggering of social tipping points, or self-reinforcing and nonlinear changes in collective behavior patterns ${ }^{14-16}$. Social tipping points, in turn, require tapping into the various feedback-loops that trigger selfreinforcing cultural evolution (as elaborated below) ${ }^{14}$.

Social tipping points will likely not be reached as long as strong countervailing forces exist. To pinpoint a few barriers, unsustainable modes of collective behavior are entrenched in social norms and habits 14,17, ecologically harmful behaviors are reinforced by existing infrastructures, technological path-dependencies, institutional lock-ins, and incentive systems ${ }^{14,18,19}$, and good intentions are impeded by structural, political, and economic factors ${ }^{18,20}$. Despite much recent work around social tipping points in sustainable behaviors ${ }^{14-16}$, there is still a considerable gap in our understanding of how these barriers may be overcome and how social tipping points may be hastened through practical interventions. In this work, we particularly focus on the barrier of infrastructure and material environments, and how they can be leveraged towards sustainable outcomes through the practice of design.

The quest for social tipping points and collective behavior change reinforces the existing notion in environmental psychology that individual-level behavior may not be the most appropriate level for sustainable behavioral interventions. For example, critical research in environmental psychology has noted that there is a general tendency in behavioral research to study individualistic and short- 
term actions with relatively low potential for greenhouse gas emission reduction ${ }^{21,22}$. Focusing only on individual cognitions, motivations or behaviors is insufficient when considering the full scope of social and contextual factors that enable or inhibit the emergence of pro-environmental behaviors 4,14 . For example, the role of the material and physical environment is often underappreciated when it comes to understanding behavior patterns - if suitable affordances (Box 1) or opportunities for sustainable behavior do not exist, sustainable behaviors will rarely emerge 14,18,23. Furthermore, research has emphasized that whilst behavior change is so often studied at the individual or household level, humans may be more effective at driving social change when working at the levels of social groups, tightly-knit social clusters, or communities ${ }^{24-27}$.

We therefore amplify the growing movement in environmental psychological sciences which seek to accommodate rapid social, cultural or community-level change as a prime target of interest for interventions ${ }^{24,25}$ and move beyond a mere individualistic focus ${ }^{21}$ when designing for social change. We argue that the fields of design and experimental architecture can support these objectives in facilitating collective behavior change, particularly by emphasizing the understudied physical and material factors that influence the emergence of sustainable behaviors $5,14,18,28,29$.

In this article, we propose ways forward in developing strategic design interventions for triggering collective sustainable behavior change, particularly within urban contexts. We do so by drawing on hands-on experiences from 15 years of work at the internationally renowned studio for visual art and architecture, RAAAF (Rietveld Architecture-Art-Affordances). RAAAF's multidisciplinary approach operates at the intersection of architecture, visual art, and philosophy. We do not argue for design as a silver bullet for sustainability concerns - certainly, many economic, political and social determinants for behavior exist that are outside the scope of design and architecture - but rather (and more humbly) intend to share lessons learned from practical experience for developing unconventional and imaginative material interventions. This is a response to a recent discussion in sustainability science emphasizing that collaborative efforts between behavioral scientists, artists and designers are required for identifying high-leverage points to intervene in sustainable behavior interventions ${ }^{5,30}$.

In the context of sustainable behaviors and design, we argue for thinking and acting strategically. Strategic design interventions, as defined in this paper and in RAAAF's work, seek to set collective social change in motion, intervene with behaviors where they matter the most, and enhance the development of skillsets for sustainable cultural evolution. Strategic design interventions develop sustainable affordances (Box 1) and dismantle unsustainable constraints, as well as develop inspiring material models for behavior intervention. Strategic design interventions situate interventions within a broader context of social change, helping collectives of active citizens to develop sustainable skillsets, imagine alternative realities that can evoke a desire for change in people, as well as inspire change in policies. 


\section{Box 1. Affordances and urban behavior.}

By the term affordance, originally a concept developed by James J. Gibson in the field of ecological psychology ${ }^{69,70}$, we refer to opportunities for action provided by the environment to people equipped with cultural skillsets ${ }^{36}$. For example, a bicycle lane constitutes an affordance of "bicycling" to a person with the appropriate culturally acquired skills of bicycling. Affordances for sustainable behavior form a central part of strategic design interventions for sustainability. The strategic design of sustainable affordances not only results in individual engagement with sustainable behaviors (and subsequent learning and skill-development), but also promotes social learning and cooperation when others observe (through teaching, copying, etc.) this behavior in a shared "landscape of affordances" 36.

Crucially, the design of affordances can have effects that much outlive individual behaviors. A bicycle lane, for example, not only affords individual behavior (by providing suitable conditions for bicycling), since this behavior is perceived and copied by others. The increased visibility of bicycling may attract further cyclers, which may increase political demand for better and safer cycling infrastructure or hasten the evolution of pro-bicycling norms or traditions (e.g., the higher visibility of cyclists or social pressure may alert cars to drive more carefully). Arguably, such development has preceded the rapid evolution of bicycling cultures in cities such as Amsterdam or Copenhagen ${ }^{14}$ - to the extent that the latter is sometimes colloquially referred to as the "City of Cyclists" ${ }^{41}$. Bicycle traffic crossing the city center of Copenhagen has approximately doubled since $1970^{14}$.

Pictured below: functional cycling infrastructure will "afford" bicycling for a person with the culturally acquired skills for bicycling. Well-designed bicycling affordances can have radical effects on the social uptake of bicycling ${ }^{14}$. Such is the case with Amsterdam, which has invested considerably into bicycling infrastructure and thus facilitated the development of its nowfamous bicycling culture. 

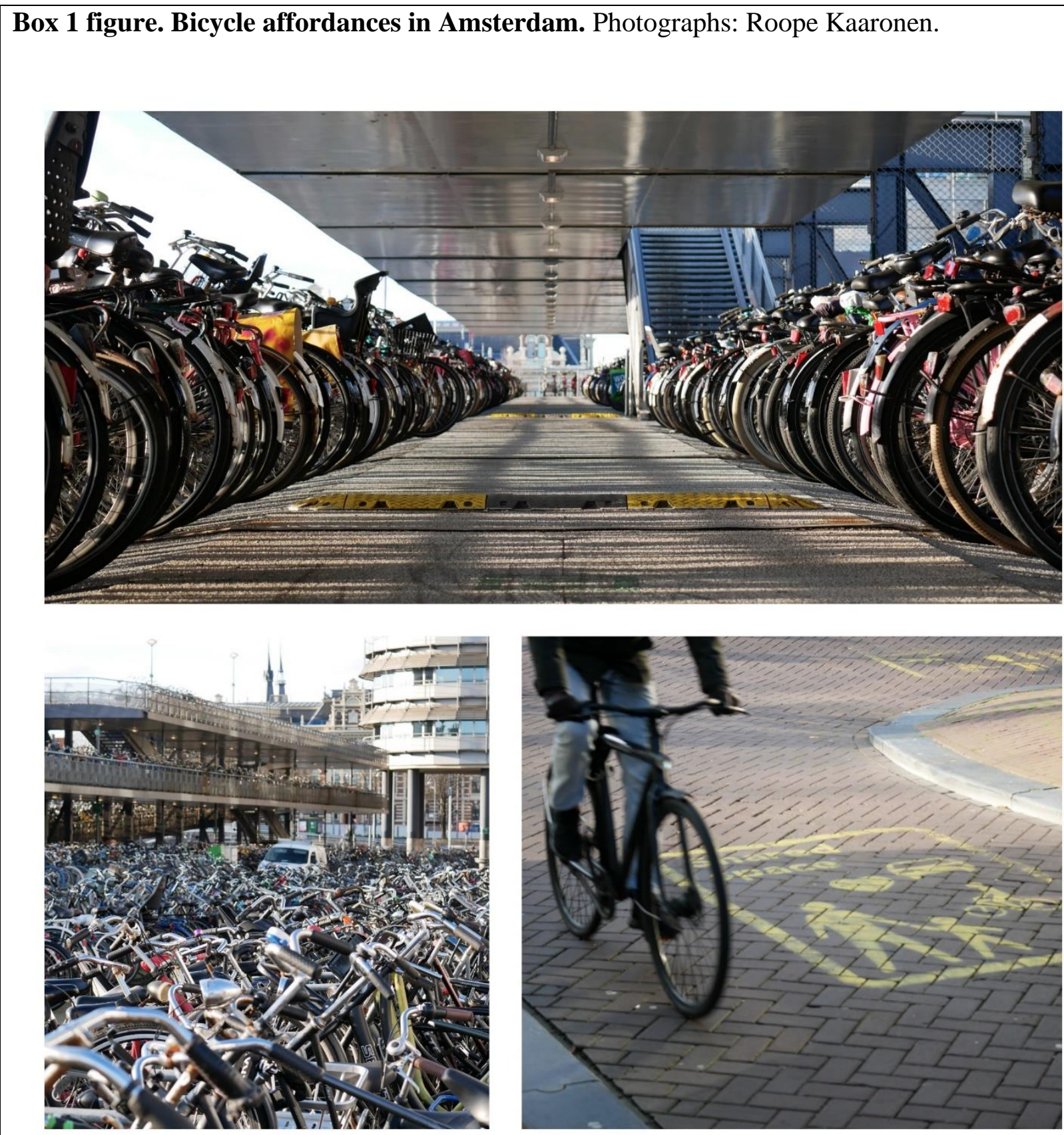

In this article, we begin by defining the idea of strategic design interventions and assess how it compares to some common design or behavior intervention approaches. We then sum up 10 key lessons for developing strategic design interventions for sustainable collective behavior change. These lessons are born from a synthesis between practice and theory: by merging insights from behavioral science and practical experiences at RAAAF, we gather pragmatic examples for developing design interventions that set social change in motion. 
We note that these lessons emerge predominantly from a Western European perspective, since most of RAAAF's projects are based in The Netherlands. This focus on a Western and industrial perspective is deemed relevant, since it is well-documented that wealthier societies tend to have higher per capita emissions, and therefore the pressure for behavior change is also arguably more pronounced $^{3,31}$. For example, one recent study ${ }^{32}$ notes that the highest income decile of the world population is responsible for ca. 34\% of household-related direct and indirect carbon emissions. However, we remain optimistic that the lessons presented below are applicable, relevant, and inspiring in other contexts as well. We do not portray these lessons as universally applicable prescriptions - rather, by reflecting upon experience at RAAAF and the challenges faced, we hope to convey an inspirational message which behavioral scientists, designers, practitioners and policymakers alike may find useful.

\section{Strategic design interventions}

Strategic design interventions, as defined by RAAAF, are "precisely chosen and carefully designed interventions in a city or landscape that set a desired development in motion." 33 By combining aspects of behavioral sciences, arts, and practical crafts (especially architecture and design), strategic design interventions adhere to three main principles, which can be summed up as follows:

1. Strategic design interventions aim to set societal change in motion.

2. Strategic design interventions seek not only to design affordances for sustainable behavior, but also to remove (and create) constraints where necessary.

3. Strategic design interventions seek to intervene where it matters the most. Often this means inspiring new ways of living or influencing new or established policy measures.

In the following, before moving onto practical lessons for designing strategic design interventions, we briefly discuss why these principles are important and how they can complement or inspire existing traditions in design or behavioral interventions.

\section{Setting social change in motion: The ecology of design}

Although we define urbanization as a major challenge for sustainability, we also perceive ample possibilities in urbanization, since urban environments unleash the potential for impactful strategic design interventions. The fact that more humans than ever before live in shared everyday environments allows us to tap into the features that most characterize human social life: our cultural capacities to intentionally engage in the design of our everyday environments (also known as "cultural niche construction"), as well as engage in social learning, skill-development, and cooperation ${ }^{34,35}$.

Previously, we have proposed ecological approaches to human behavior ${ }^{14,33,36-39}$ which emphasize the various self-reinforcing feedback-loops that drive cultural processes and have the potential for 
triggering social tipping points. These can be summed up in what we call the Ecology of Design 14,38,39 (Figure 1):

- Humans inhabit a rich landscape of affordances, which provides behavioral opportunities relative to our cultural forms of life (see Box 1).

- Any behaviors solicited by these affordances can be spread socially through social learning (e.g., through conformism or the copying or teaching of behaviors in social networks).

- Behaviors solicited by the affordances within our environment can reinforce learned behaviors through skill-development, habituation, and other forms of individual learning.

- These affordances can further be altered and refined through intentional cultural niche construction (i.e., design of everyday environments and infrastructure).

Figure 1. Ecology of Design. Illustrating the various feedback-loops of the Ecology of Design. Landscapes of affordances afford behaviors; behaviors alter affordances (and can dismantle/develop constraints); behaviors spread and are learned socially; behaviors lead to and are reinforced by individual learning and habituation.

\section{Ecology of Design}

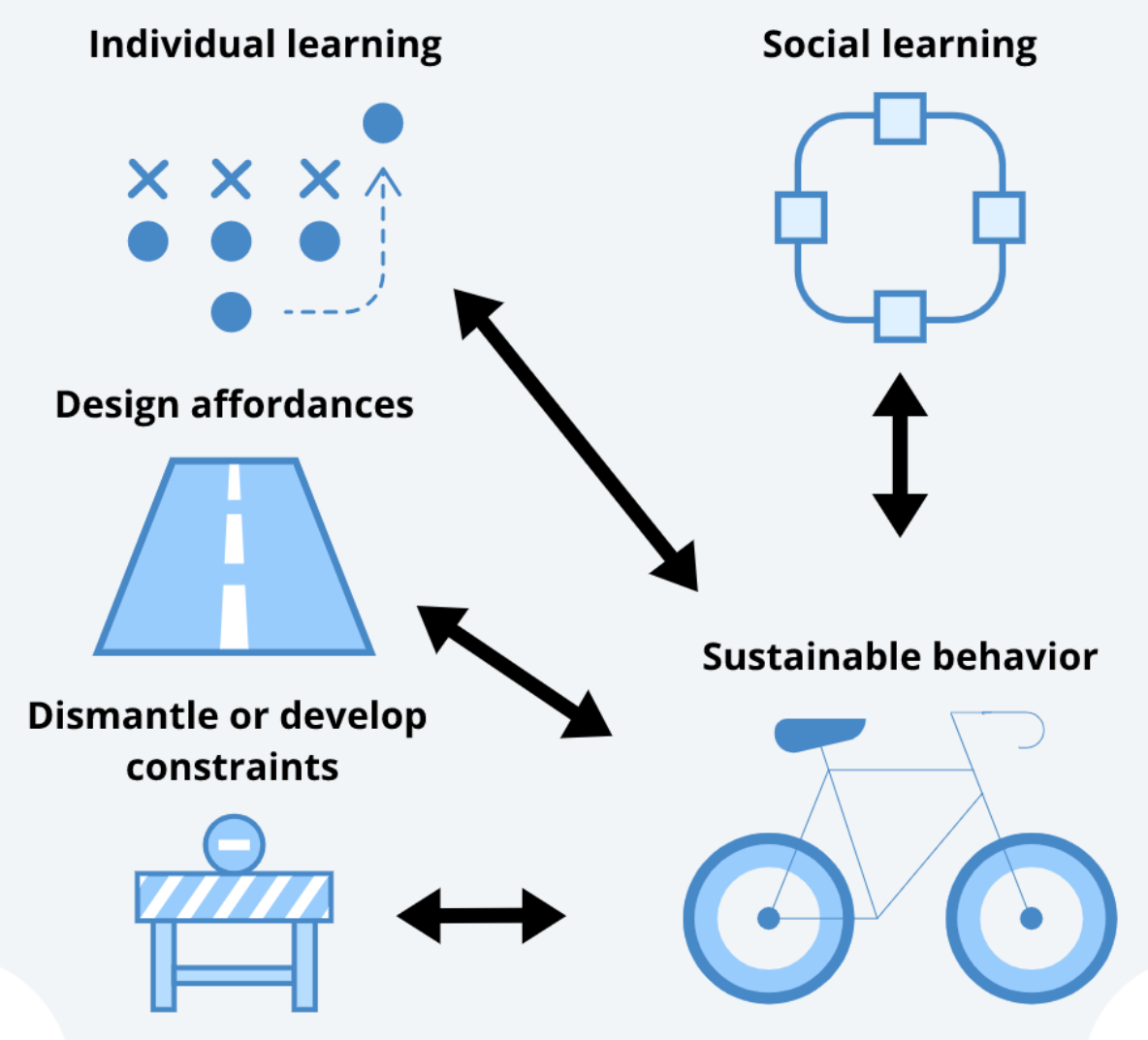


These four feedback-loops defined above together suggest that the design of everyday environments can have longer consequences and ripple effects than often presumed (see also Box 1). Indeed, simulation studies ${ }^{14}$ have illustrated that under such assumptions, it can be expected that even a linear or steady increase of affordances for sustainable behavior over time can produce nonlinear tipping points in the collective adoption of sustainable behavior patterns. This is evident, for example, in cities such as Copenhagen or Amsterdam, which have facilitated the rapid and accelerating adoption of bicycling behaviors by the gradual design of improved cycling infrastructure (Box 1) ${ }^{14,41}$.

By aiming to set social change in motion, the target of strategic design interventions is not as much changing individual behaviors as it is changing sociocultural practices. Accordingly, we wish to distinguish strategic design interventions from the movement in industrial design literature going under the name of "design for sustainable behavior" ${ }^{42,43}$. In doing so, we are not arguing that strategic design interventions should replace (or otherwise compete with) industrial or product design - they can form complementary approaches.

In line with proponents of "design for sustainable behavior", we argue that behavior change research should take the practice or art of design more seriously. "Design for sustainable behavior" is important in its own right, because it emphasizes:

1. convenient pro-environmental user-interaction with products ${ }^{43}$,

2. sustainable life-cycles of products ${ }^{44}$, and/or,

3. the design of environmentally friendly environments and contexts ${ }^{42,45}$.

However, our approach to strategic design underlines that "design" should not merely involve the sustainable design of products and their lifecycles. These approaches place less emphasis on the social learning or skill-development that follows the design intervention, or the cultural practices that ensue. With proponents of critical design approaches ${ }^{46}$, we agree that sociocultural practices should form the basic target for design, and that facilitating social transitions should be the main objective of design intervention.

We would thus like to emphasize that the notion of design interventions could be of greater relevance for sustainability if it would shift its focus from individual behaviors to sociocultural practices. As described below, strategic design interventions seek to set desired collective behavior patterns in motion. In such a context, a "sustainable behavior" should not be understood merely as an endpoint, but rather as a trigger of subsequent social processes (as depicted in Figure 1).

\section{Designing affordances and removing constraints}

Strategic design interventions seek not only to design affordances for sustainable behavior, but also to 1. remove constraints for desirable behaviors and 2. create constraints for undesirable behaviors. Constraints are to be understood as features of the environment that either prohibit or 
impede a behavior. This implies that strategic design interventions diverge from some prevalent frameworks for behavioral or design interventions in important ways.

For one, it is notable that some influential trends in design interventions, such as those under the broad umbrella of "nudging", are wary of designing constraints. Although nudge theory is a relative newcomer to the field of behavior interventions, it has already made a mark as one of the most influential approaches to studying behavior change in the $21^{\text {st }}$ century, not only shaping academic research but also governmental policies worldwide. Nudge theory emphasizes that design interventions should not reduce the liberties of those nudged ${ }^{47}$. In doing so, it suggests that the redesign of environments (or "choice architectures") should alter "people's behavior in a predictable way without forbidding any options" or reducing the liberties of those nudged. By definition, a nudge should not constrain behaviors. In practice, however, this is often difficult to achieve as predictable behavior change might require the design of environments that, in all pragmatic terms, constrain options. As we illustrate below with case studies, in the case of challenging entrenched unsustainable habits, it will often be necessary to design constraints for unwished-for behaviors.

Second, in its libertarian desire to not reduce the freedoms of those nudged, nudge theory generally disregards the notion of positive freedoms: many behavioral interventions and alterations of behavior settings can in fact increase liberties by designing entirely new landscapes of affordances that allow previously inaccessible alternative forms of behavior ${ }^{38}$. Moreover, sometimes interventions can increase liberties by removing existing environmental barriers and constraints ${ }^{33}$. These are also core targets of strategic design interventions, as illustrated with case studies below.

\section{Intervene where it matters the most in the long run}

For developing strategic design approaches, we draw inspiration from research in "strategic education" 48,49 . Strategic environmental education begins by asking "what kinds of actions most effectively address environmental problems" ${ }^{48}$. Notably, whilst individual behavior change is necessary in the transitions ahead, focus on private and individual behaviors alone is insufficient for meeting sustainability targets. For one example, in the EU in 2018, households represented only $26 \%$ of final energy consumption, or $17 \%$ of gross inland energy consumption ${ }^{50}$. Privatesphere interventions therefore have limited scope, and next to changing behaviors of citizens, strategic design interventions should 1. emphasize active citizenship and organizing for collective public change ${ }^{48}, 2$. inspire new or existing policies to raise their ambitions in meeting sustainability targets, and 3. collaborate with practical experts, stakeholders and industry to materialize visions of alternative futures. Accordingly, strategic design intervention aim to shift the focus from the individual private-sphere to collective, communal and prosocial processes $24,25,33$. 
Strategic design interventions therefore seek to respond to some recent concerned voices in environmental psychology who suggest that prevailing behavioral or design interventions are too individualistic and thus not particularly well-suited for triggering social change on the scale currently required ${ }^{21,24,25}$. It is not unreasonable to argue that a significant portion of environmental behavioral science and psychology has traditionally had an issue with measuring what counts the most ${ }^{22}$. Recent critical work in environmental psychology has highlighted how research in the field has measured behavioral outcomes that are ecologically, politically or emissions-wise, far from the most important and strategic ones ${ }^{21,22}$. For instance, many popular surveys in environmental psychology still include near-trivial items such as "I always switch the light off when I don't need it on anymore" 51 . Furthermore, too often research in environmental behavior focuses on low-cost behaviors, such as cheap individual consumption choices. Yet evidently, as noted above, sustainability transitions will require high-cost behavior change and behaviors aligned against existing traditions. Necessary high-cost behaviors include social activism ${ }^{52}$, giving up deeply entrenched habits, or moving against well-established social conventions, norms and infrastructural barriers. It is important to ask how high-cost behaviors such as these might emerge 26,27 .

Strategic design interventions should therefore shift the focus to exploring and developing those behaviors that can make impact by promoting sustainability in the long run. Moreover, strategic design interventions seek to grow sustainable behaviors in everyday context and situations. This implies a shift from studying behavioral outcomes, determinants, or predecessors to the more practical task of triggering collective behavioral processes ${ }^{34}$. The emphasis here is on the notion that to study how sustainable cultures may emerge in urban environments, we must immerse ourselves in their development and self-organization, as outlined by $20^{\text {th }}$ century urban critics such as Jane Jacobs ${ }^{53}$ and Christopher Alexander ${ }^{54,55}$.

Moreover, in recent discussion on the contribution of behavioral science to sustainability concerns, it has also been repeatedly proposed that to ensure longer-term impact researchers should form alliances with practical professionals $5,21,56$. Indeed, professionals such as artisans, engineers, architects, and urban designers have valuable reservoirs of practical realism and expertise to guide the redesign of our social and material everyday environments. Strategic design interventions, being born from a synthesis of practical and theoretical knowledge, seek to employ practical knowledge by developing explorative interventions in-situ and in realistic context, forming alliances between practitioners and researchers in the process.

These new alliances should also encompass policymakers. As illustrated below with case studies, thanks to their imaginative power and by selecting target audiences with care, strategic design interventions can also directly influence even national-level policies, driving social change at a scale where design or behavioral interventions do not typically reach. Strategic design interventions can be inspirational, helping both decision-makers and the broader public explore and imagine alternative futures ${ }^{5}$. The notion of inspiring policy change by presenting alternative future living environments is even more important due to the fact that changing public behaviors 
is often a slow process (even the aforementioned development of "bicycling cultures" has taken a couple of decades ${ }^{14,41}$ ), and therefore directly influencing local or national policies is important to help reach the ambitious timescales needed for sustainability transitions.

\section{Lessons for strategic design interventions}

In recent discussion, the role of solutions-oriented practical knowledge in behavioral science and sustainability transitions has been emphasized ${ }^{5,56,57}$. Accordingly, in the following, we draw on practical experiences from RAAAF ${ }^{58}$, a collective that makes imaginative interventions at the cross roads of visual art, architecture and philosophy. We outline 10 lessons learned for designing strategic design interventions based on RAAAF's 15 years of practical experience. We also discuss some challenges encountered along the way, and how they might be overcome. The following lessons are based partly on interviews with RAAAF members Ronald Rietveld and David Habets.

Using select case studies, we target these lessons for researchers, practitioners, and policymakers alike. The case studies were selected from RAAAF's portfolio of projects ${ }^{58,33}$. In doing so, we do not claim that these experiences are prescriptive or directly imitable models for designing strategic interventions for sustainable behavior. Nor do we claim that all the selected case studies represent all features of strategic design interventions as defined above, or that they are applicable universally. Our goal is humbler: to inspire scientific researchers and policy makers to collaborate with practitioners to develop ambitious material design interventions that have the capacity for setting sustainable social change in motion. The following provides practical lessons to help accomplish this.

\section{The designer's own fascinations and ambitions}

One possible starting point for strategic design interventions are the designer's fascinations and ambitions for developing a design. For designers and artists this may seem obvious, but perhaps less so for behavioral scientists. Designing an intervention that sets social change in motion cannot disregard experience and phenomenology: for an intervention to feel "alive" ${ }^{54}$, it must take seriously the experiential, material and aesthetic component of everyday life, including local context. This implies a shift in the researcher from a passive observer to an active and passionate designer. The role of passion, although often disregarded in scientific experimentation, cannot be understated, since the design and implementation of strategic interventions require sustained care and adaptation over the course of many years, given their trial-and-error nature. Many contemporary concerns - particularly issues related to sustainability - can feel overwhelming. Maintaining one's personal interest and energy-levels high over the course of many years is therefore of particular importance. Notably, this also does not prohibit strategic interventions from having a social or political agenda: in the current context of socio-ecological crises, researchers 
may drive social change on a scale that could be defined as "political" 59 , particularly when promoting equity, sustainability, and setting desired developments in motion.

\section{Analysis on different scale levels of a site's existing qualities}

Designing a successful strategic intervention requires deep knowledge of not only the architectural or infrastructural realities, but also of local natural, material, cultural, historical and social qualities. This includes the intervention site's location within knowledge networks ${ }^{60}$. This implies that to set social change in motion, designs or behavior interventions cannot merely impose uniform "nudges" or designs on people, but require more elaborate responses respectful of local qualities. Recall that affordances (Box 1) are relational, and therefore the same physical environment will not provide the same opportunities for behavior for all people or cultures. Affordances are always contingent on local capabilities ${ }^{38}$. There is always much information at local level interactions that is lost in top-down impositions of design: for example, a local population may not wish to be imposed upon, and may instead wish to be helped in achieving their common goals.

Indeed, much of our capacity to solve problems rests with the "informal web of creative and regulatory relationships we have" in our cultural interactions ${ }^{54}$. Strategic design interventions therefore subscribe to ideals such as polycentricity ${ }^{61}$ in governance, which respects that decisionmaking should have many local and context-aware centers, as well as the principle of subsidiarity, the notion that interventions should be designed close to the target audience.

Analyzing a site's existing qualities is, for example, a driving principle in RAAAF's planned Trusted Strangers intervention ${ }^{62}$ (Box 2), which seeks to imaginatively design social affordances that align with 1. the historical qualities of water city Amsterdam and 2. that are sensitive to the cultural diversity of the city, with the specific aim of promoting social cohesion - and therefore,

social sustainability and resilience ${ }^{63}$ - in urban environments. Here, the specific aim is to set positive social change in motion and connect seemingly conflicting interests by designing affordances that encourage prosocial behaviors and cultural interactions. 


\section{Box 2. Trusted Strangers.}

The city of Amsterdam will celebrate its $750^{\text {th }}$ anniversary in 2025. Amsterdam is known for its liberal culture, and the city's ambition is to celebrate this heritage for its anniversary. RAAAF responded to the cities ambition, as well as to its urgent need for good public domain in and around Amsterdam North, with the project Trusted Strangers.

Trusted Strangers is a planned strategic intervention with the intention of promoting social cohesion in Amsterdam ${ }^{62}$. It would dock a fleet of barges along the northern bank of the River IJ, by a former dockyard that overlooks Amsterdam's historical city across the water. A grid of 24 large barges (each $80 \mathrm{~m}$ long, $11 \mathrm{~m}$ wide and $6 \mathrm{~m}$ high) would enclose a social world with an abundance of social affordances for various subcultures in each barge (e.g., skateparks and birdwatchers), but also include barges that have affordances for more widely appreciated affordances (e.g., campfires and a football pitch) that promote positive interactions between the various attracted subcultures. The focus on water is aligned with Amsterdam's history and culture, which have always shaped the form and culture of the city.

By affording unexpected encounters between various cultural groups, the aim of the intervention is to develop physical affordances that help coinhabitants of Amsterdam develop into "trusted familiar strangers", increasing social cohesion and thus social sustainability. The issue of social cohesion is crucial in the increasingly segregated and polarized Western societies. For more information, see Rietveld et al. ${ }^{62}$ and RAAAF ${ }^{58}$. A video is also available at https://vimeo.com/205663543 (password: welcomestranger).

Box 2 figure. Trusted Strangers by RAAAF. Photo: ArtefactoryLab, Olivier Campagne / RAAAF. Reprinted with permission.

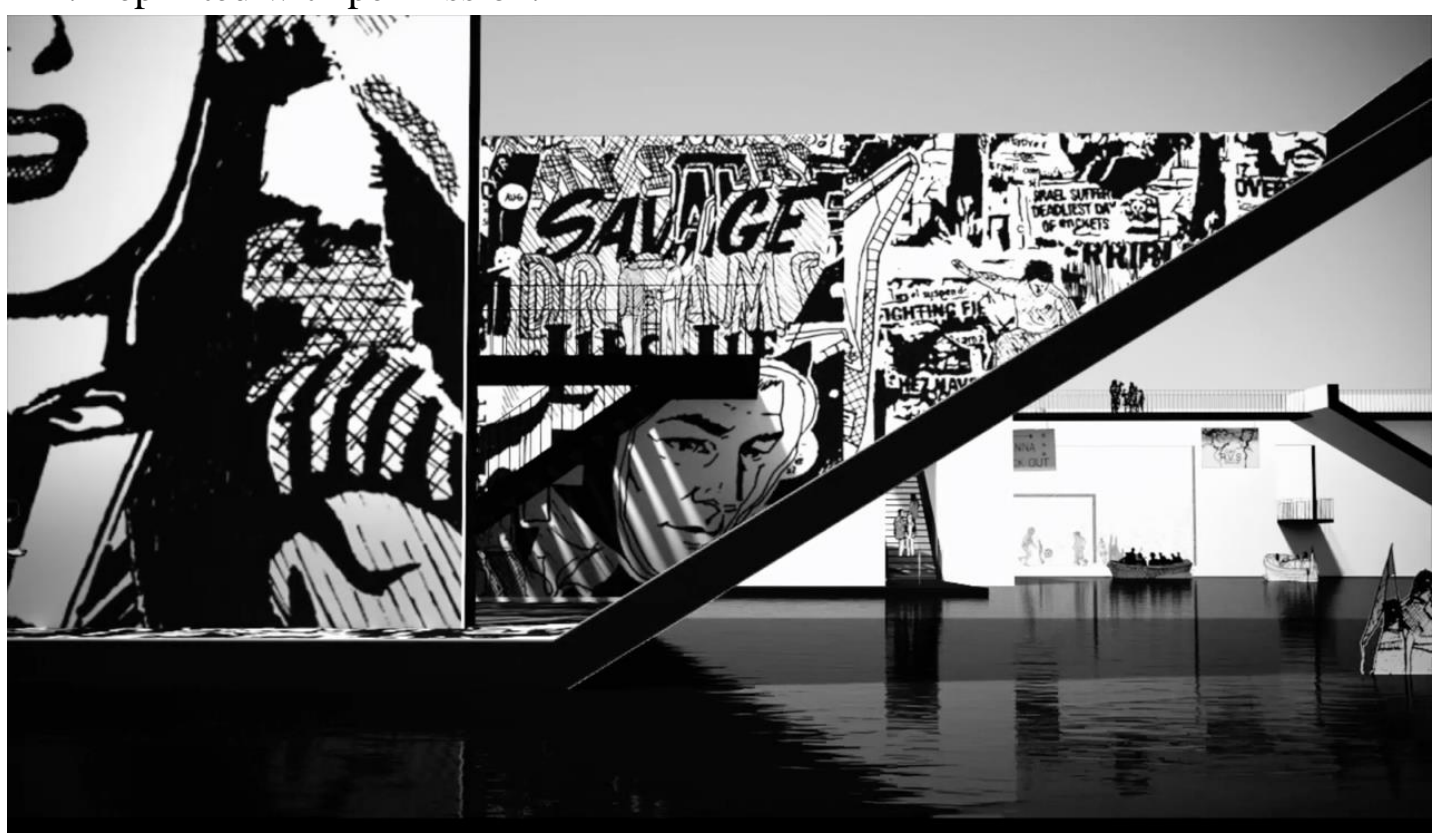




\section{Use the forces of large-scale developments and processes}

Strategic design interventions seek to locate interventions in contexts and situations where they align with larger-scale forces in development and socio-political processes. Accounting for largescale social, infrastructural and political developments is important if a design is wished to have impact where it matters to most in the long run and set social development in motion. This is important since design alone will not solve the problem of collective sustainable behavior change. Therefore, RAAAF's interventions seek to inspire and align with existing policies, legislation, and local informal rules and norms ${ }^{61}$. Indeed, we argue below that by tapping into existing political realities and structures, strategic design interventions best serve when they complement and coexist with other large scale social and institutional change.

For example, RAAAF has specifically targeted the use of vacancy for innovating with design experiments ${ }^{33}$. In RAAAF's Dutch contribution to the Venice Biennale 2010, Vacant $N L$, the potential of 10,000 governmentally-owned vacant buildings was illustrated (Box 3) ${ }^{58}$, connecting RAAAF's design interventions with large-scale developments in vacancy. Vacancy is not only a waste of public resources and space, but also has considerable consequences for social and ecological sustainability: the carbon emissions of building new infrastructures could considerably be reduced by using vacant spaces more efficiently (e.g., cement accounts for ca. $8 \%$ of global $\mathrm{CO} 2$ emissions ${ }^{64}$, not to mention other carbon-intensive construction materials such as steel). Moreover, vacancy interferes directly with the social fabric that maintains lively and cohesive societies ${ }^{53,54}$. By targeting policy-makers as a key target audience, RAAAF was able to directly inspire national vacancy policies and, consequently, advance the use of vacant space in the Netherlands (Box 3). This illustrates how strategic design interventions can directly inspire impactful policies.

In later interventions, RAAAF has more specifically sought to make use of vacancy and intervene with it in ways that connect conflicting interests ${ }_{-}^{33}$ (Box 5). Vacancy will likely be an even larger issue in the coming years due COVID-19 and its consequent socioeconomic crises which have seen the closing down of public buildings worldwide. Note also that temporary re-use of public vacant places (e.g. churches, gymnasia, airbases) could rapidly increase the amount of available public space in crowded cities to accommodate for, e.g., the social distancing needs of pandemics.

Many challenges exist when trying to align projects with large-scale developments. For one, the responsibility for taking the initiative is often left to the artist or designer. Typically, few funding instruments exist that seek artistic or design input for large-scale projects. Moreover, commissioners in architecture and urban design are rarely seeking for work whose output is not strictly pre-determined, which may considerably reduce the degrees of freedom for creative and unconventional interventions. Often, the possibility of actually realizing a project is determined by merely "being at the right place at the right time" - serendipitously meeting suitable stakeholders and funding instruments during the course of persistent practice. 


\section{Box 3. Vacant NL.}

Vacant NL illustrates the potential of 10.000 government-owned vacant buildings from the 17th20th centuries. In Vacant NL, the Dutch contribution to the Venice Biennale 2010, RAAAF built miniature models of these vacant buildings from blue foam and presented them in the otherwise empty Gerrit Rietveld pavilion, which had itself been vacant for 39 years for seven months a year. The pavilion itself thus became part of the total installation. The installation highlights the architectural waste that characterizes most modern cities today, and calls attention to the unsustainable use of urban infrastructure by means of visual presentation. Vacant NL is a part of RAAAF's broader interest in studying vacancy. For more information, see Rietveld et al. ${ }^{33}$ and RAAAF ${ }^{58}$.

Vacant $N L$ was partly commissioned by the Ministry of Education, Culture and Science. Vacant $N L$ inspired the launch of a Master Program at The Sandberg Institute (Rietveld Art Academy, Amsterdam), as well as two books: one documenting the totality of vacant public buildings in the Netherlands ${ }^{60}$, and one the opportunities and challenges of using vacant space in architecture 71. Together, these left a mark on Dutch policies for making use of vacant public infrastructure: since then, the Dutch Chief Government Architect has made re-use of vacancy a top priority.

Box 3 figure. Vacant $N L$ by RAAAF. Photo: Rob 't Hart. Reprinted with permission.

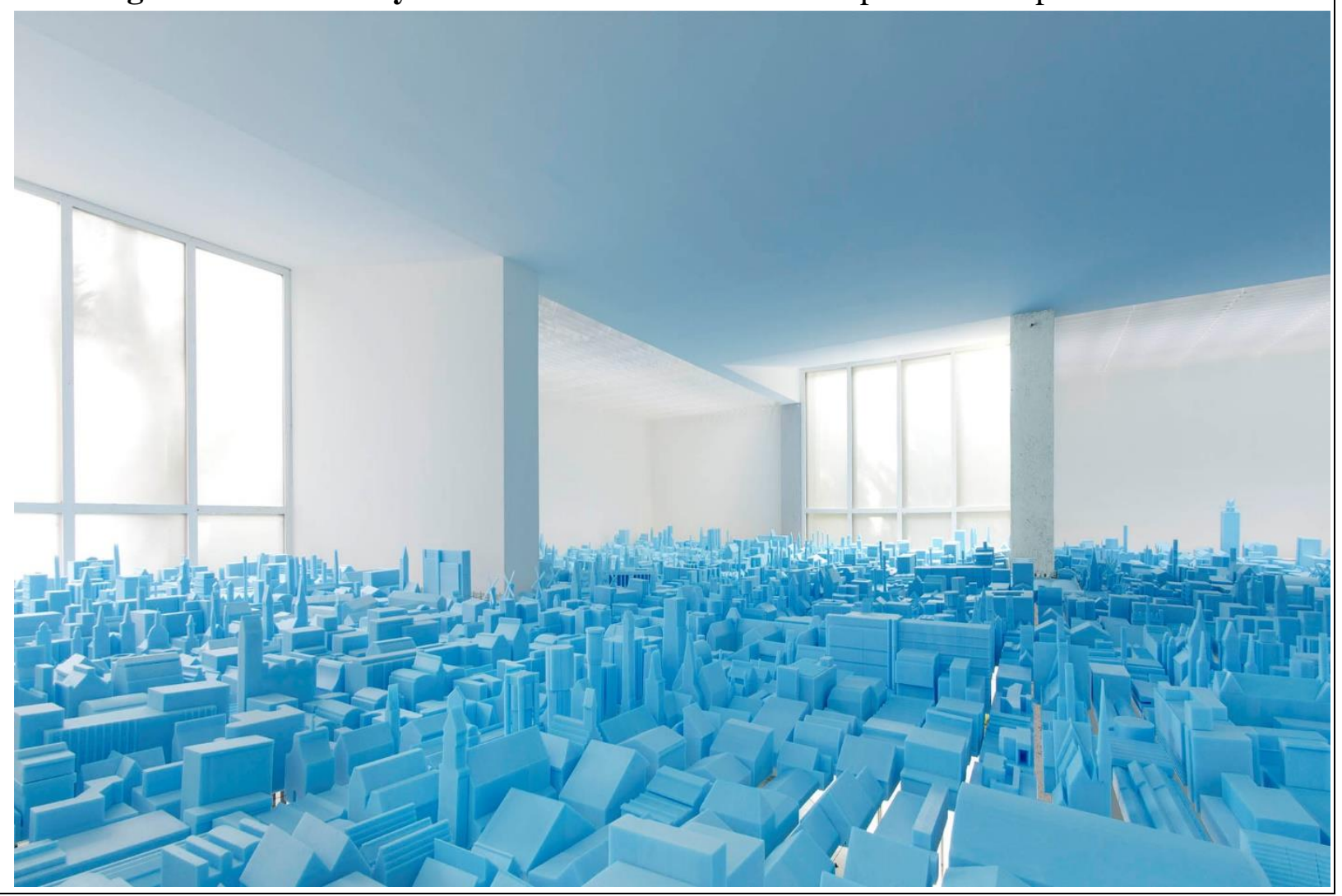




\section{New alliances with researchers, interested parties and specialists}

If designers or behavioral scientists wish to make relevant contributions to addressing large-scale social or ecological issues, the complexity of these concerns dictate that it is necessary to forge alliances with researchers, stakeholders, practitioners and other specialists. This aligns well with the ample discussion on utilizing various forms of knowledge in sustainability science ${ }^{56}$. All too often the behavioral research disregards the practical experiences of artisans, (urban) designers, architects, engineers and other professions that have accumulated decades or sometimes centuries of experience in dealing with human behavior in practice. These fields represent rich pools of expertise to draw from when dealing with sustainability concerns. New alliances are particularly important, because they aid in identifying what might be relevant for the particular location as well as relevant large scale developments one's intervention could surf on.

For example, RAAAF was invited to join the recently established "COVID-19 \& Mental Resilience Expert Group" set up and chaired by the Mayor of Amsterdam. For its contribution to the expert-group RAAAF explores how the temporary re-use of vacant government and public buildings can increase people's mental and emotional well-being by creating new affordances for social interaction in novel, socially-distanced ways.

Forming new alliances can, however, be challenging. One particularly valuable skill is to learn to tell "tailored" stories to gain the attention of various interest groups. A strategic design intervention is often multifaceted and can be framed in multiple ways: Vacant $N L$ (Box 3), for instance, could be framed in ways which highlight environmental sustainability (by reducing the need to construct new buildings due to re-use of vacancy), social sustainability (by, e.g., creating new avenues for social activities, skill-sharing or cultural exchange in underutilized buildings), or economic sustainability (by providing affordable real-estate to, e.g., artists and young scientists, in otherwise neglected properties).

New alliances can also involve the combination of various methodological approaches. Whilst strategic design interventions offer deep qualitative scope on how behavior change develops, their effects can also be measured quantitatively ${ }^{65}$. For instance, in an interdisciplinary collaboration, RAAAF's The End of Sitting (Box 4) afforded movement scientists with the opportunity to empirically study how alternative working environments prevent sedentary behavior ${ }^{65}$.

\section{Box 4. The End of Sitting.}

The End of Sitting is an experimental installation "at the crossroads of visual art, architecture, philosophy and empirical science" designed in collaboration between RAAAF and Barbara Visser ${ }^{58}$. Acknowledging that prolonged sitting has detrimental health effects and therefore a negative effect on sustainable well-being ${ }^{65}$, RAAAF sought to provide a new vision on the landscape of affordances (see Box 1) for office spaces of the future. The affordances in modern offices not only promote the behavior of sitting, but also constrain other forms of more active 
postures. The affordances in The End of Sitting, contrarily, both enable various active positions for supported standing and constrain sitting.

Accounting for various body shapes and sizes and through interactive and participatory design processes, RAAAF designed a new landscape of office affordances which "consist of a landscape of inclined planes to support different standing and leaning positions" and promote the user's active exploration of new positions for working. The installation's variety of affordances solicit visitors of various capabilities to explore alternatives to sitting postures in an experimental office landscape. In the open office landscape, participants are also able to observe how others are working in the non-sitting positions offered by the space and learn socially.

The End of Sitting can be considered the start of an experimental trial phase, exploring the possibilities for radical change in working environments. The End of Sitting also presented avenues for empirical research for movement scientists ${ }^{65}$, who found that the new office "supported the well-being of participants more so than a conventional office" and "had no negative effects on reported concentration levels and satisfaction with the produced work." By means of expert meetings, The End of Sitting also targeted policy-makers directly in its audience, aiming to inspire policy change through its temporary installation. For more information, see Rietveld ${ }^{72}$, Caljouw et al. ${ }^{65}$ and RAAAF ${ }^{58}$.

Box 4 figure. The End of Sitting by RAAAF | Barbara Visser. Photo: Jan Kempenaers. Reprinted with permission.

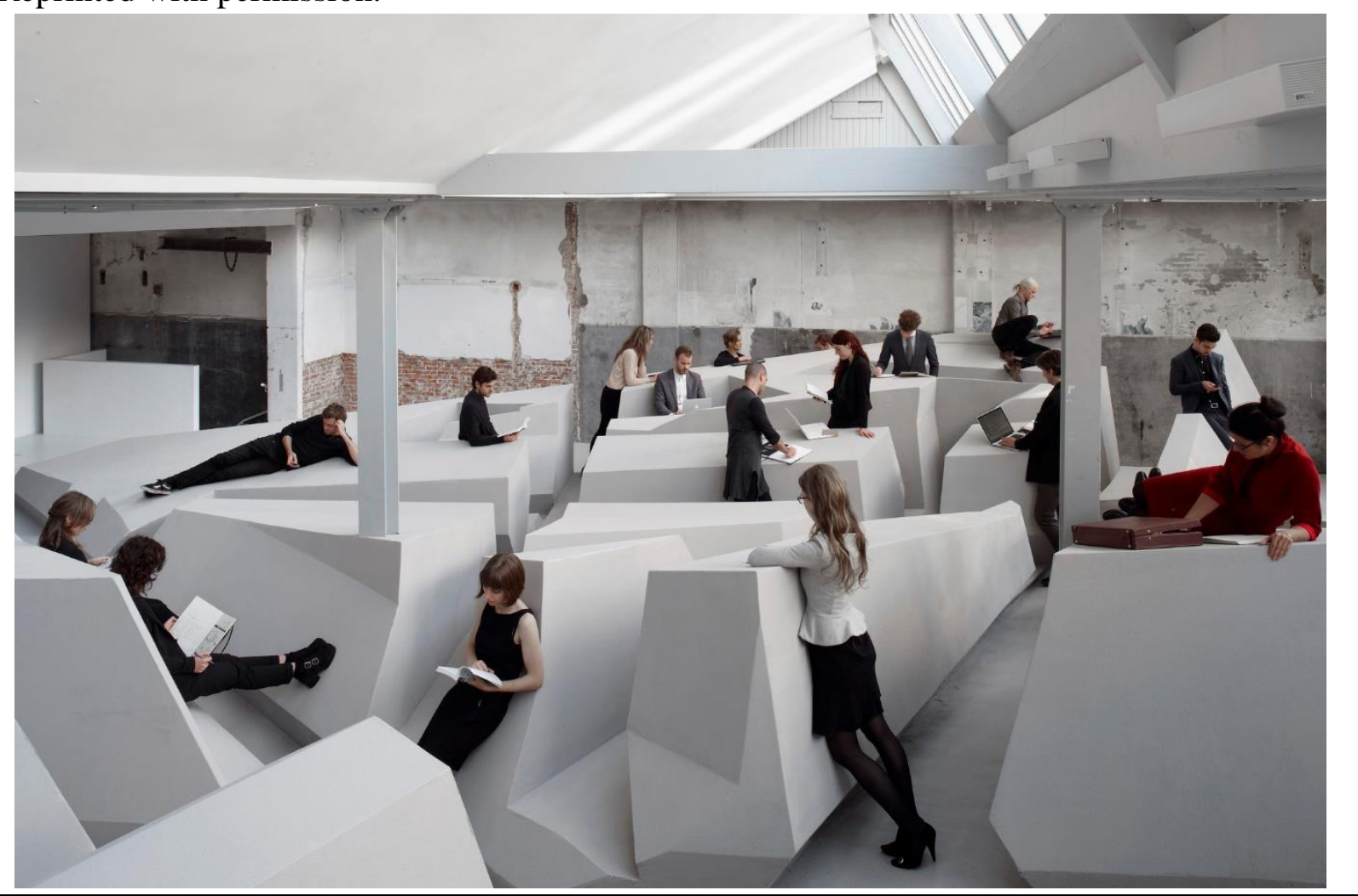




\section{Setting desired developments in motion}

Strategic design interventions emphasize that the realization of a design is not the end, but rather a starting point for the development or evolution of a new social practice or set of skills. The idea of "design as the end state" has been inherent in much of modernist philosophy, urban design and architecture ${ }^{54}$. However, this need not be the case, and we propose that the product of a successful strategic design intervention should merely be the starting point of the further development of collective sustainable behavior patterns and cultures. The targets of strategic design interventions are therefore not individual behaviors as such, but sociocultural practices. Accordingly, interventions are best located in contexts where they can facilitate the development of skills and encourage social behavior change. Strategic design interventions seek to accomplish this by making sustainable behaviors easy and salient, also encouraging the active exploration of new, sustainable affordances (see Boxes 2 and 4). Here we refer back to the discussion on the Ecology of Design: design can have ripple effects that are much stronger than often imagined, as illustrated by the effects of urban design on the development of bicycling cultures ${ }^{14}$.

This is partly evident in RAAAF's experimental installation The End of Sitting (Box 4), whose design setting allows for participants to observe each other and actively learn new skillsets to explore their new environment, setting social change in motion. Although The End of Sitting is only in its experimental trial phase, its core findings and ideas can subsequently be developed to promote alternative office cultures. Note also how the approach differs from the philosophy of nudging (see, e.g., ${ }^{66}$ for an office-based nudge). Instead of seeking to covertly "nudge" participants, The End of Sitting 1. reduced constraints for alternative working postures, 2. afforded the active exploration and discovery of multiple new, personally convenient working postures, 3 . created constraints for sitting behaviors, and 4. inspired participants to reflect upon their entrenched sitting habits and learn new ones. We propose that to achieve long-term behavior change there exists little reason why change in behavior should be merely "nudged" instead of offering entirely new landscapes of affordances.

Humans are notoriously habitual, and changing entrenched habits can be particularly challenging to overcome. For instance, if chairs are still available in the vicinity of a The End of Sitting installation, people often revert to their old sitting habits. This relates to our earlier critique of nudge-approaches: often, to inspire true change in collective behaviors, simple iterations of the status quo may be insufficient, and more total redesigns of everyday environments are required.

Setting social change in motion is, however, a long-span process. Often the results of strategic design interventions are not initially visible. Therefore, their effects would also be difficult to study with traditional methods in behavioral science. It is important to also understand strategic design interventions as using imagination for "planting seeds" from which alternative realities may grow from. For example, an experience of an installation such as The End of Sitting may spark the desire for an alternative way of living. This "planting of seeds" can also involve placing well-thought 
themes and concepts into public discussion and political agendas (such as with Vacant NL, Box 3), reinforcing existing streams of social change.

\section{Connecting seemingly conflicting interests and issues}

Typically, a variety of interests and issues play a role in a design project. This is particularly the case with sustainability concerns, which often hide conflicting or even diametrically opposed interests (e.g., differences in costs and benefits between groups with respect to environmental, social, or economic outcomes). Strategic design interventions should identify such conflicts through analysis of cultural and historical qualities.

Sometimes, however, a radical solution is devisable in which contradictory interests can be solved with mutual benefit. Take, for example, Bunker 599, a Second World War bunker with burdened heritage, which RAAAF sliced in two in a strategic design intervention (Box 5). Here, conflicting interests lied between heritage preservation, dealing with burdened heritage, and opening historical monuments to public use. Typically, historical infrastructure and cultural heritage is preserved and safeguarded. However, this is problematic when dealing with burdened heritage, since preserving and safeguarding historically burdened monuments can also send an unwished-for message, as international protests against colonialist statues have made manifest in 2020. By slicing one monument open (Bunker 599), the remaining bunkers in The Netherlands gained significance in the public's eye. Following the intervention, Bunker 599 became a Dutch national heritage site. Therefore, strategic design interventions can set a precedent for dealing creatively with burdened history and increase the cultural significance of built cultural heritage. Through attentive design, there might be a way to connect conflicts of interests.

One particularly challenging tension is that between strategic design interventions and regulatory environments. Unconventional environments such as The End of Sitting, Bunker 599 or Trusted Strangers often come with safety concerns: who is responsible for an unlikely, but still possible, accident? This is a larger problem in urban design, where many bold reimaginations of everyday environments so often hit regulatory barriers. With strategic design interventions, there are some ways to circumvent the problem. One is to present the intervention strictly as visual art, with appropriate caution signs and guides. However, this leads to obvious challenges with regards to scalability: art exhibitions cannot typically be scaled to large-scale urban contexts. Another way is to design environments and the affordances within in ways which considerably mitigate risks of accidents. However, each case highlights the notion that large-scale social change will be difficult to achieve without the guidance and cooperation of regulatory institutions and experts, and perhaps even a change in the risk-avoidant behavior of governments. 


\section{Box 5. Bunker 599.}

In the installation Bunker 599, RAAAF, in collaboration with Atelier de Lyon, sliced open a seemingly indestructible WWII-era concrete bunker. In doing so, it radically reinterpreted Dutch and UNESCO policies on cultural heritage. By slicing and opening the interior of one The Netherlands' WWII-era bunkers (which are typically cut off from view completely), it created new meaning to interpreting the approximately 700 others. Furthermore, a long wooden boardwalk takes the visitor through the bunker, leading the visitor to an adjacent natural reserve, itself a product of wartime alterations.

Dealing with burdened history (such as WWII history in The Netherlands) is an important part of cultural and social sustainability. Bunker 599 illustrated how burdened cultural heritage can be dealt with creatively, opening new affordances for cultural interaction through redesign of existing monuments. It is now a publicly accessible attraction for visitors, visible from the A2 highway and therefore seen by tens of thousands of daily passers-by. Paradoxically, following the cutting intervention Bunker 599 became a Dutch national monument, shedding new light on Dutch and UNESCO policies on cultural heritage. The Dutch Cultural Heritage Agency now uses Bunker 599 to illustrate how creative alterations can increase the cultural significance of historically burdened environments. For further information, see Rietveld et al. ${ }^{33}$ and RAAAF. For a video, see https://vimeo.com/213978423.

Box 5 figure. Bunker 599 by RAAAF | Atelier de Lyon. Photo: Allard Bovenberg. Reprinted with permission.

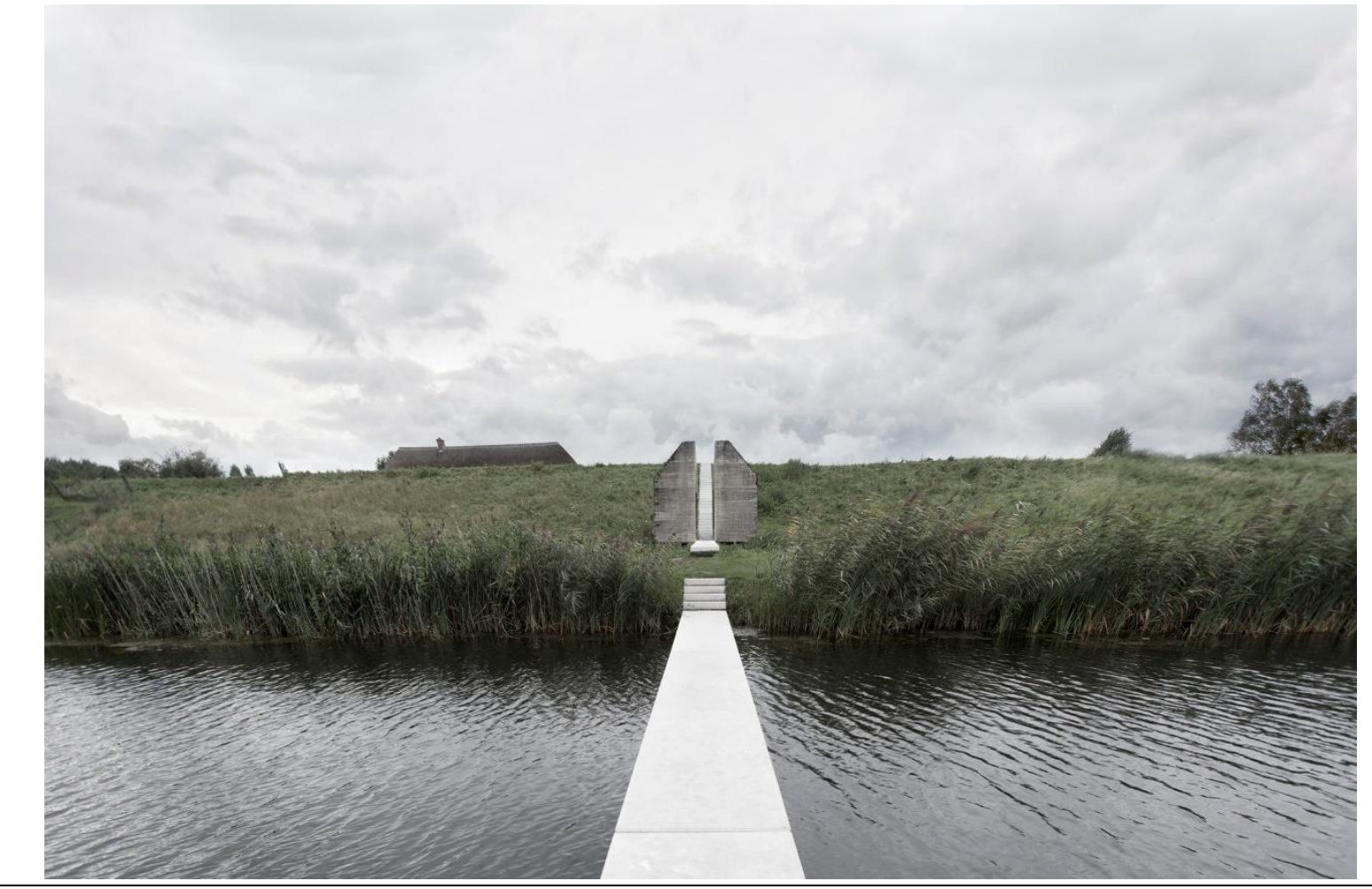




\section{Extending long-term ambitions}

As outlined above, many traditional behavior interventions are not particularly well-suited for tackling the sustainability challenges defined in the introduction, since they are short-term and local interventions that do not scale. Meanwhile, the most impactful opportunities for sustainable social change are often found at a larger scale level (such as municipal, regional, national or international policy) and connect with long-term ambitions on time scales spanning decades. Sustainability is about sustaining over time, and short-term interventions disconnected from largescale developments simply won't suffice ${ }^{5}$. Strategic design interventions should therefore create sufficient opportunities for long-lasting spin-offs, and should ask how to forge alliances by looking at a longer timescale for overlapping interests. Should an intervention be successful, it should be possible to extend it over the long-term. This aligns with strategic design intervention's ambition to not only observe or measure social behaviors but also grow them. Therefore, strategic design interventions should envision an intervention's or site's long-term significance in order to move towards this vision when opportunity arises. Particularly important here is to acknowledge potential alliances with researchers, stakeholders, experts and other interested parties: when designing strategic interventions, one might ask what organizations, social groups, programs, businesses or parties would be willing to continue the development after the intervention's initial phase.

\section{Connecting scale levels}

Even when design interventions are implemented in the smaller scale levels (e.g., intervene with local practices), the designer must be aware of developments on the larger scale levels, including local, municipal, national and international development. To extend long-term ambitions and use the force of large-scale developments, it is important to link up with or capitalize on their policies and visions. When changing a design, the designer should consider the impact of the intervention on other (larger and smaller) scale levels. It has been well documented that design should be connected with its context, to ensure that the intervention is both relevant and functional ${ }^{54}$. Consequently, strategic design interventions can also seek to evolve material environments in "structure preserving transformations" 54 - transformations that do not "reinvent" design but rather alter existing structures (e.g., Bunker 599, Box 5), maintaining harmony between different levels of scale ${ }^{54}$. This also underlies RAAAF's interest with vacancy (Box 3): rather than doing away with vacant buildings and thus "reinventing" architecture (as is often done in modernist urban planning ${ }^{54}$ ), RAAAF's interventions have sought to evolve vacant structures to more socially desirable forms ${ }^{33}$. 


\section{Design for spontaneity}

Strategic interventions can also leave enough "wiggle room" for spontaneous use to emerge later. Acknowledging that humans are adept at learning social and cultural skills also implies that their collective behavior patterns will portray spontaneity and self-organizing patterns, which are typically highly unpredictable ${ }^{54}$. Strategic design interventions can therefore involve the conscious design of spontaneous interactions. Instead of pre-programming an intended use, strategic design interventions can embrace the spontaneity and unpredictability of human social life. Indeed, this spontaneity and unpredictability is arguably one of the core creative forces underlying the success of cities more generally ${ }^{54}$. Through temporary interventions, strategic design interventions can also push people out of habitual "comfort zones" (see, e.g., The End of Sitting, Box 4), encouraging them to explore spontaneous behaviors and reflect upon their entrenched habits. Such temporary experimentation can, when successful, also lead to long-term developments.

For another example, the strategic design intervention Trusted Strangers (Box 2) creates urban affordances for various cultures and subcultures in ways that afford interactions between social or cultural groups, and purposely leaves room for social groups to spontaneously interact and learn from each other. In the long run, such interactions can result in knowledge spillover, encouraging the exchange of ideas, cultural skillsets and so on, increasing social cohesion and social sustainability ${ }^{54}$. Similarly, whilst The End of Sitting (Box 4) has the provocative intention of providing alternatives for sitting, it leaves plenty of degrees of freedom for spontaneous, unpredictable use of the various affordances for working positions.

\section{Clear and powerful concept and visualization}

Finally, the articulated and visualized concept of a strategic design intervention should be clear and powerful. We argue that human aesthetic experiences are too often disregarded in behavioral interventions, and making sustainable interventions and lifestyles aesthetically attractive is particularly important, given that sustainable alternatives are often perceived as second-grade or associated with "giving up" aesthetic preferences ${ }^{67}$. After all, it has been well established since the work of John Dewey in the 1930s, that art and aesthetics play a major role in education, learning, and skill-development ${ }^{68}$.

Strategic design interventions should therefore improve on the aesthetic design of behavior interventions. This can be supported in various ways. For example, in the process of making, one can use sketches, 3D drawings, scale models, full-scale mock-ups, or hone a concept. Ideally one uses all these in tandem. We therefore propose increased cooperation between researchers and those with practical knowledge and experience in various forms of art and design. 


\section{Conclusion}

In this article, we have proposed that to better align with the challenges of the Anthropocene, behavior interventions should be designed more strategically, with the specific aims of 1. changing sociocultural practices and setting social change in motion, 2. designing affordances and removing constraints for sustainable behaviors, and 3. intervening with behaviors that matter the most, which often means interacting with policy-makers and stimulating their imagination. To achieve this, combining knowledge from various practical professions and crafts with behavioral science will be useful. To inspire the implementation of strategic design interventions, we gathered 10 practical lessons for designing strategic design interventions for sustainable behavior change by the experimental architecture studio RAAAF. Whereas scientific studies aim to describe reality as it is, RAAAF's material interventions help exploring how the shared living environment could be in the future. We hope the practical lessons we have drawn from this will inspire ambitious design and behavioral interventions.

\section{Acknowledgments}

We are grateful to the people at RAAAF, especially Ronald Rietveld and David Habets, for sharing their expertise in their work. We also thank Julian Kiverstein, Jelle Bruineberg, Jasper van den Herik, Ludger van Dijk, Maarten van Westen, Twan Kieboom, and Harry Heft for commenting on earlier versions of this paper, and two anonymous reviewers for their insightful suggestions. Roope

Kaaronen received funding from the Doctoral Programme in Social Sciences at the University of Helsinki and the Netherlands Organization for Scientific Research (NWO, VIDI grant, 276-20019). Erik Rietveld is supported by the Netherlands Organization for Scientific Research (NWO, VIDI grant, 276-20-019) and the European Research Council (ERC Starting Grant, 679190). 


\section{References}

1. TWI2050 (2019). Transformations to Achieve the Sustainable Development Goals. Report prepared by The World in 2050 initiative (International Institute for Applied Systems Analysis (IIASA)).

2. Steffen, W., Richardson, K., Rockström, J., Cornell, S.E., Fetzer, I., Bennett, E.M., Biggs, R., Carpenter, S.R., De Vries, W., and De Wit, C.A. (2015). Planetary boundaries: Guiding human development on a changing planet. Science $347,1259855$.

3. Steffen, W., Broadgate, W., Deutsch, L., Gaffney, O., and Ludwig, C. (2015). The trajectory of the Anthropocene: The Great Acceleration. The Anthropocene Review 2, 81-98.

4. Steg, L. (2018). Limiting climate change requires research on climate action. Nature Climate Change 8,759-761.

5. Lotz, L., Pickering, J., Weber, E., Contestabile, M., Pearson, A., Voinov, A., Townsend, A., Ebers, A., Zeeuw van der Laan, A., and Fischhoff, B. (2019). Twenty questions about design behavior for sustainability, report of the International Expert Panel on behavioral science for design. Nature Sustainability.

6. United Nations, Department of Economic and Social Affairs, Population Division (2019). World Urbanization Prospects: The 2018 Revision (ST/ESA/SER.A/420) (United Nations).

7. West, G.B. (2017). Scale: The Universal Laws of Growth, Innovation, Sustainability, and the Pace of Life in Organisms, Cities, Economies, and Companies (Penguin).

8. Seto, K.C., Güneralp, B., and Hutyra, L.R. (2012). Global forecasts of urban expansion to 2030 and direct impacts on biodiversity and carbon pools. PNAS 109, 16083-16088.

9. Solecki, W., Rosenzweig, C., Dhakal, S., Roberts, D., Barau, A.S., Schultz, S., and ÜrgeVorsatz, D. (2018). City transformations in a $1.5^{\circ} \mathrm{C}$ warmer world. Nature Clim Change 8 , 177-181.

10. McPhearson, T., M. Raymond, C., Gulsrud, N., Albert, C., Coles, N., Fagerholm, N., Nagatsu, M., Olafsson, A.S., Soininen, N., and Vierikko, K. (2021). Radical changes are needed for transformations to a good Anthropocene. npj Urban Sustain 1, 1-13.

11. Raworth, K. (2017). Doughnut Economics: Seven Ways to Think Like a 21st-Century Economist (Chelsea Green Publishing).

12. Sadorsky, P. (2018). Shifts in energy consumption driven by urbanization. The Oxford Handbook of Energy and Society, 179.

13. Lenton, T.M., Rockström, J., Gaffney, O., Rahmstorf, S., Richardson, K., Steffen, W., and Schellnhuber, H.J. (2019). Climate tipping points - too risky to bet against. Nature 575, 592595. 
14. Kaaronen, R.O., and Strelkovskii, N. (2020). Cultural evolution of sustainable behaviors: Proenvironmental tipping points in an agent-based model. One Earth 2, 85-97.

15. Otto, I.M., Donges, J.F., Cremades, R., Bhowmik, A., Hewitt, R.J., Lucht, W., Rockström, J., Allerberger, F., McCaffrey, M., and Doe, S.S. (2020). Social tipping dynamics for stabilizing Earth's climate by 2050. Proceedings of the National Academy of Sciences 117, 2354-2365.

16. Milkoreit, M., Hodbod, J., Baggio, J., Benessaiah, K., Calderón-Contreras, R., Donges, J.F., Mathias, J.-D., Rocha, J.C., Schoon, M., and Werners, S.E. (2018). Defining tipping points for social-ecological systems scholarship — an interdisciplinary literature review. Environ. Res. Lett. 13.

17. Nyborg, K., Anderies, J.M., Dannenberg, A., Lindahl, T., Schill, C., Schlüter, M., Adger, W.N., Arrow, K.J., Barrett, S., and Carpenter, S. (2016). Social norms as solutions. Science $354,42-43$.

18. Kaaronen, R.O. (2017). Affording sustainability: Adopting a theory of affordances as a guiding heuristic for environmental policy. Front. Psychol. 8.

19. Bolderdijk, J.W., and Steg, L. (2015). Promoting sustainable consumption: The risks of using financial incentives. In Handbook of research on sustainable consumption (Edward Elgar Publishing).

20. Moser, S., and Kleinhückelkotten, S. (2018). Good Intents, but Low Impacts: Diverging Importance of Motivational and Socioeconomic Determinants Explaining Pro-Environmental Behavior, Energy Use, and Carbon Footprint. Environment and Behavior 50, 626-656.

21. Nielsen, K.S., Clayton, S., Stern, P.C., Dietz, T., Capstick, S., and Whitmarsh, L. (2020). How psychology can help limit climate change. American Psychologist.

22. Huddart Kennedy, E., Krahn, H., and Krogman, N.T. (2015). Are we counting what counts? A closer look at environmental concern, pro-environmental behaviour, and carbon footprint. Local Environment 20, 220-236.

23. Marcus, L., Giusti, M., and Barthel, S. (2016). Cognitive affordances in sustainable urbanism: contributions of space syntax and spatial cognition. Journal of Urban Design 21, 439-452.

24. Bamberg, S., Rees, J.H., and Schulte, M. (2018). Environmental protection through societal change: What psychology knows about collective climate action-and what it needs to find out. In Psychology and Climate Change (Elsevier), pp. 185-213.

25. Gausset, Q. (2019). Stronger together: How Danish environmental communities influence behavioural and societal changes. In The Role of Non-State Actors in the Green Transition: Building a Sustainable Future, pp. 52-70.

26. Centola, D. (2018). How Behavior Spreads: The Science of Complex Contagions (Princeton University Press). 
27. Centola, D. (2021). Change: How to Make Big Things Happen (New York City, NY: Little, Brown and Company).

28. Steg, L., and Vlek, C. (2009). Encouraging pro-environmental behaviour: An integrative review and research agenda. Journal of Environmental Psychology 29, 309-317.

29. Rietveld, E. (2019). The affordances of art for making technologies. Inaugural lecture at the University of Twente, Socrates Chair.

30. Round-up on design behaviour (2019). Nature Sustainability 2, 1065-1065.

31. Kartha, S., Kemp-Benedict, E., Ghosh, E., Nazareth, A., and Gore, T. (2020). The Carbon Inequality Era: An assessment of the global distribution of consumption emissions among individuals from 1990 to 2015 and beyond.

32. Hubacek, K., Baiocchi, G., Feng, K., Muñoz Castillo, R., Sun, L., and Xue, J. (2017). Global carbon inequality. Energ. Ecol. Environ. 2, 361-369.

33. Rietveld, E., Rietveld, R., and Mackic, A. (2014). Strategic interventions as a design approach $=$ Strategische interventies als ontwerpbenadering. In Vacancy Studies: Experiments \& Strategic Interventions in Architecture $=$ Experimenten \& Strategische Interventies in Architectuur (nai010 Publishers), pp. 79-116.

34. Henrich, J. (2015). The Secret of our Success: How Culture is Driving Human Evolution, Domesticating our Species, and Making us Smarter (Princeton University Press).

35. Laland, K.N. (2018). Darwin's Unfinished Symphony: How Culture Made the Human Mind (Princeton University Press).

36. Rietveld, E., and Kiverstein, J. (2014). A rich landscape of affordances. Ecological Psychology $26,325-352$.

37. Kiverstein, J., van Dijk, L., and Rietveld, E. (2019). The field and landscape of affordances: Koffka's two environments revisited. Synthese.

38. Kaaronen, R.O. (2020). Steps to a Sustainable Mind: Explorations into the Ecology of Mind and Behaviour. (Publications of the Faculty of Social Sciences, University of Helsinki).

39. Kaaronen, R.O. (2019). The Ecology of Design. In The Side View Journal (The Side View Press), pp. 17-21.

40. Tennie, C., Call, J., and Tomasello, M. (2009). Ratcheting up the ratchet: On the evolution of cumulative culture. Philos. Trans. R. Soc. Lond., B, Biol. Sci. 364, 2405-2415.

41. Gössling, S. (2013). Urban transport transitions: Copenhagen, City of Cyclists. Journal of Transport Geography 33, 196-206. 
42. Lockton, D., Harrison, D., and Stanton, N. (2008). Making the user more efficient: Design for sustainable behaviour. International Journal of Sustainable Engineering 1, 3-8.

43. Wever, R., Van Kuijk, J., and Boks, C. (2008). User-centred design for sustainable behaviour. International Journal of Sustainable Engineering 1, 9-20.

44. Ramani, K., Ramanujan, D., Bernstein, W.Z., Zhao, F., Sutherland, J., Handwerker, C., Choi, J.-K., Kim, H., and Thurston, D. (2010). Integrated sustainable life cycle design: A review. J. Mech. Des 132.

45. Lockton, D. (2012). Affordances, constraints and information flows as "leverage points" in design for sustainable behaviour (Social Science Research Network).

46. Mazé, R., Gregory, J., and Redström, J. (2011). Social sustainability: A design research approach to sustainable development. 4th World Conference on Design Research (IASDR).

47. Thaler, R.H., and Sunstein, C.R. (2008). Nudge: Improving Decisions about Health, Wealth, and Happiness (Yale University Press).

48. Chawla, L., and Cushing, D.F. (2007). Education for strategic environmental behavior. Environmental Education Research 13, 437-452.

49. Heft, H., and Chawla, L. (2006). Children as agents in sustainable development: The ecology of competence. In Children and their Environments: Learning, Using and Designing Spaces, pp. 199-216.

50. EuroStat (2020). Energy consumption in households - Statistics Explained. https://ec.europa.eu/eurostat/statistics-

explained/index.php/Energy_consumption_in_households.

51. Milfont, T.L., and Duckitt, J. (2010). The environmental attitudes inventory: A valid and reliable measure to assess the structure of environmental attitudes. Journal of Environmental Psychology 30, 80-94.

52. Hagedorn, G., Kalmus, P., Mann, M., Vicca, S., Berge, J.V. den, Ypersele, J.-P. van, Bourg, D., Rotmans, J., Kaaronen, R., Rahmstorf, S., et al. (2019). Concerns of young protesters are justified. Science 364, 139-140.

53. Jacobs, J. (1961). The Death and Life of Great American Cities (Random House).

54. Mehaffy, M.W. (2017). Cities Alive: Jane Jacobs, Christopher Alexander, and the Roots of the New Urban Renaissance (Sustasis Press).

55. Alexander, C. (1979). The Timeless Way of Building (Oxford University Press).

56. Caniglia, G., Luederitz, C., Wirth, T. von, Fazey, I., Martín-López, B., Hondrila, K., König, A., Wehrden, H. von, Schäpke, N.A., Laubichler, M.D., et al. (2020). A pluralistic and 
integrated approach to action-oriented knowledge for sustainability. Nature Sustainability, 18 .

57. Watts, D.J. (2017). Should social science be more solution-oriented? Nature Human Behaviour $1,1-5$.

58. RAAAF https://www.raaaf.nl/.

59. Why Nature needs to cover politics now more than ever (2020). Nature 586, 169-170.

60. Rietveld, R., and Rietveld, E. (2010). Dutch Atlas of Vacancy (With Graphic Sesign by Studio Joost Grootens). (NAI).

61. Ostrom, E. (2010). Beyond markets and states: Polycentric governance of complex economic systems. American Economic Review 100, 641-72.

62. Rietveld, E., Rietveld, R., and Martens, J. (2017). Trusted strangers: Social affordances for social cohesion. Phenomenology and the Cognitive Sciences.

63. Aldrich, D.P., and Meyer, M.A. (2015). Social Capital and Community Resilience. American Behavioral Scientist 59, 254-269.

64. Lehne, J., and Preston, F. (2018). Making concrete change: Innovation in low-carbon cement and concrete. Chatham House Report, Energy Enivronment and Resources Department: London, UK, 1-66.

65. Caljouw, S.R., de Vries, R., and Withagen, R. (2017). RAAAF's office landscape The End of Sitting: Energy expenditure and temporary comfort when working in non-sitting postures. PloS one $12, \mathrm{e} 0187529$.

66. Venema, T.A.G., Kroese, F.M., and Ridder, D.T.D.D. (2018). I'm still standing: A longitudinal study on the effect of a default nudge. Psychology \& Health 33, 669-681.

67. Luchs, M.G., and Kumar, M. (2017). "Yes, but this other one looks better/works better": How do consumers respond to trade-offs between sustainability and other valued attributes? J Bus Ethics 140, 567-584.

68. Uhrmacher, P.B. (2009). Toward a theory of aesthetic learning experiences. Curriculum Inquiry $39,613-636$.

69. Gibson, J.J. (1979). The Ecological Approach to Visual Perception (Houghton Mifflin).

70. Heft, H. (2001). Ecological Psychology in Context: James Gibson, Roger Barker, and the Legacy of William James's Radical Empiricism (Psychology Press).

71. Rietveld, R. (2014). Vacancy studies: Experiments \& strategic interventions in architecture (nai010 publishers). 
72. Rietveld, E. (2016). Situating the embodied mind in a landscape of standing affordances for living without chairs: materializing a philosophical worldview. Sports Medicine 46, 927-932. 